[0212-7199 (2006) 23: 7; pp 338-344] ANALES DE MEDICINA INTERNA Copyright (C) 2006 ARAN EDICIONES, S.L.

AN MED INTERNA (Madrid) Vol. 23, N. ${ }^{\circ}$ 7, pp. 338-344, 2006

\section{Efectos adversos de los fármacos antirretrovirales. Fisiopatología, manifestaciones clínicas y tratamiento}

\author{
E. SANTOS CORRALIZA, A. FUERTES MARTÍN
}

Servicio de Medicina Interna II. Hospital Universitario. Salamanca

\section{RESUMEN}

La disminución plasmática de la carga vírica de pacientes con infección por el VIH, por debajo de los límites de cuantificación de las técnicas más sensibles disponibles en la actualidad, junto con el aumento del número de linfocitos $\mathrm{CD} 4$, se ha asociado con un descenso en el riesgo de padecer enfermedades oportunistas en estos pacientes. El descenso de la morbilidad y la mortalidad, es especialmente importante desde la introducción en 1996, del tratamiento antirretrovírico de gran actividad (TARGA). Sin embargo, el tratamiento prolongado con estas combinaciones de fármacos puede ser dificultosos debido a los problemas que generan la adherencia y los efectos adversos. Todos los antirretrovirales pueden producir efectos secundarios a corto y largo plazo. El riesgo de efectos adversos específicos varía según el fármaco, la clase a la que pertenece e incluso la susceptibilidad individual. El mejor conocimiento de los efectos adversos de los fármacos antirretrovirales es necesario para intentar optimizar el tratamiento en pacientes infectados por el VIH.

PALABRAS CLAVE: Fármacos antirretrovirales. Factores adversos.
SIDE EFFECTS OF ANTIRETROVIRAL THERAPY. FISIOPATHOLOGY, CLINICAL MANIFESTATIONS AND TREATMENT

\begin{abstract}
The suppression of plasma viral loads in HIV infected patients to less than the limit of quantification of the most sensitive commercially available assays and the coincident improvement in CD4 T cell counts is associated with a decrease in the risk of new opportunistic infections in this patients. The descent at the morbibility and mortality is specialy important since the introduction in 1996 the hard activity antirretroviral therapy (HAART). However, prolonged treatment with combination regimens can be difficult to sustain because of problems with adherence and toxic effects. All antiretroviral drugs can have both short-term and longterm adverse events. The risk of specific side effects varies acording to specific drug, drug class and even, individual susceptibility. A better understanding of the adverse effects of antiretroviral agents is of interest to try to optimize therapy in HIV infected patients.
\end{abstract}

KEY WORDS: Antiretroviral therapy. Side effects.

Santos Corraliza E, Fuertes Martín A. Efectos adversos de los fármacos antirretrovirales. Fisiopatología, manifestaciones clínicas y tratamiento. An Med Interna (Madrid) 2006; 23: 338-344.

\section{INTRODUCCIÓN}

Desde que en 1981 se empezaron a conocer los primeros casos del síndrome de inmunodeficiencia adquirida (SIDA), los intentos de combatir la enfermedad han sido múltiples y a menudo infructuosos. Sin embargo, el mejor conocimiento de la misma, los avances en su diagnóstico y seguimiento, así como la terapia antirretrovírica, cada vez más intensa y especializada, han conseguido que la infección por el VIH pueda definirse como una enfermedad de curso crónico en los países más desarrollados. El descenso de la morbilidad y la mortalidad es especialmente espectacular desde la introducción en 1996 del tratamiento antirretrovírico de gran actividad (TARGA) $(1,2)$. Sin embargo, la utilización del TARGA asocia importantes inconvenientes que pueden disminuir considerablemente su eficacia con el tiempo, entre los que cabe destacar el desarrollo de resistencias, la dificultad en el cumplimiento terapéutico y la gran toxicidad que producen los fármacos (3).

\section{TOXICIDAD DE LOS FÁRMACOS ANTIRRETROVIRALES GENERALIDADES}

La frecuencia de la toxicidad producida por los fármacos antirretrovirales es un problema creciente en los últimos años, debido entre otras causas, a la mayor supervivencia de los

Trabajo aceptado: 15 de marzo de 2006

Correspondencia: Erika Santos Corraliza. Hospital Universitario. Servicio de Medicina Interna II. 5 ${ }^{\mathrm{a}}$ planta. Paseo de San Vicente, 58-182. 37007 Salamanca.e-mail: erikasan80@hotmail.com 
pacientes, a la necesidad de realizar tratamiento de por vida y al gran número de fármacos disponibles en la actualidad, autorizados en muchas ocasiones de forma acelerada $(3,4)$. Según un estudio suizo realizado en el año 2001, un 47\% de los pacientes tratados sufren efectos clínicos adversos y un $27 \%$ alteraciones de laboratorio, siendo el 9 y el $16 \%$ de ellos respectivamente de grado importante (5). La toxicidad es la primera causa de interrupción y modificación del TARGA, superando al fracaso virológico y a la falta de adherencia (3). En un ensayo realizado por Riesler y cols., de entre los pacientes con infección por el VIH y tratamiento antirretroviral, 11,4\% pacientes/año presentan efectos adversos grado 4 (riesgo vital). Los más frecuentes son las alteraciones hepáticas, seguidas de neutropenia, anemia y alteraciones cardiovasculares (6).

Entre los numerosos factores de riesgo analizados en relación con el desarrollo de toxicidad, los más frecuentemente encontrados son el seguir una terapia con ritonavir a dosis terapéutica y el pertenecer al sexo femenino $(7,8)$. Esto podría deberse a que las mujeres alcancen concentraciones más elevadas de fármaco en sangre, a que sean más susceptibles a los efectos tóxicos o a que comuniquen más dichos efectos que los pacientes de sexo masculino (8). Otros factores de riesgo estudiados tales como la edad, la tasa de CD4 o la carga vírica, parecen tener una menor relevancia clínica $(8,9)$.

Los efectos adversos relacionados con los fármacos antirretrovirales se pueden clasificar atendiendo a diferentes criterios:

1. Tiempo de aparición (Tabla I): precoces si aparecen dentro de los tres primeros meses tras el inicio del tratamiento (ej: hipersensibilidad) y tardíos si aparecen con posterioridad (ej: lipodistrofia).

2. Especificidad: toxicidad inespecífica producida por varios antirretrovíricos independientemente del grupo al que pertenezcan (p. ej.: gastrointestinales, hepatitis, ginecomastia) y toxicidad específica, relacionada únicamente con un determinado grupo o fármaco individual.

\section{TABLA I}

\section{EFECTOS ADVERSOS DE LOS ANTIRRETROVIRALES SEGÚN TIEMPO DE APARICIÓN}

\begin{tabular}{ll}
\hline Precoces & Tardíos \\
\hline Gastrointestinales & Daño mitocondrial \\
Hipersensibilidad & Metabólicos \\
Neuropsíquicos & Lipodistrofia \\
Hepatitis & Hepatitis \\
& Nefrolitiasis e insuficiencia renal \\
& Osteopenia y osteoporosis \\
& Necrosis ósea aséptica \\
\hline
\end{tabular}

\section{TOXICIDAD ESPECÍFICA}

Aunque a lo largo de los últimos años, ha aumentado el número de fármacos disponibles frente al VIH, el tratamiento antirretrovírico de gran actividad suele estar formado por una combinación de fármacos de tres grupos fundamentales de antirretrovirales (Tabla II): inhibidores de la transcriptasa inversa análogos de nucleósidos (ITIAN), inhibidores de la transcripta-

\begin{tabular}{llll}
\multicolumn{4}{c}{ TABLA II } \\
\hline FÁRMACOS ANTIRRETROVIRALES \\
\hline ITIAN & ITINAN & IP & IF \\
\hline Zidovudina (AZT) & Nevirapina & Saquinavir & Enfuvirtida (T20) \\
Didanosina (ddl) & Efavirenz & Nelfinavir & \\
Lamivudina (3TC) & & Ritonavir & \\
Zalcitabina (ddC) & & Indinavir & \\
Estavudina (d4T) & & Amprenavir & \\
Abacavir & & Lopinavir \\
Emtricitabina & & Atazanavir \\
ITIA nucleótidos & & Fosamprenavir \\
Tenofovir & & \\
\hline
\end{tabular}

ITIAN: inhibidores de la transcriptasa inversa análogos de nucleósidos; ITINAN: inhibidores de la transcriptasa inversa no análogos de nucleósidos; IP: inhibidores de la proteasa; IF: inhibidores de la fusión; ITIA nucleótidos: inhibidores de la transcriptasa inversa análogos de nucleótidos.

sa inversa no análogos de nucleósidos (ITINAN) e inhibidores de la proteasa (IP). Los efectos adversos específicos de cada grupo de antirretrovirales se exponen en la tabla III.

\section{TABLA III}

\section{TOXICIDAD ESPECÍFICA SEGÚN GRUPO DE} ANTIRRETROVIRALES

\begin{tabular}{|c|c|}
\hline $\begin{array}{l}\text { inversa análogos de nucleósidos } \\
\text { (ITIAN) }\end{array}$ & Toxicidad mitocondrial \\
\hline $\begin{array}{l}\text { 2. Inhibidores de la transcriptasa } \\
\text { inversa no análogos de nucleósidos } \\
\text { (ITINAN) }\end{array}$ & $\begin{array}{l}\text { Hipersensibilidad y } \\
\text { hepatotoxicidad }\end{array}$ \\
\hline $\begin{array}{l}\text { 3. Inhibidores de la proteasa } \\
\text { (IP) }\end{array}$ & $\begin{array}{l}\text { Alteraciones metabólicas } \\
\text { y lipodistrofia }\end{array}$ \\
\hline
\end{tabular}

INHIBIDORES DE LA TRANSCRIPTASA INVERSA ANÁLOGOS DE NUCLEÓSIDOS (ITIAN)

En cuanto a la toxicidad específica de los ITIAN, es necesario destacar la producción de daño mitocondrial (Tabla IV). Las mitocondrias son los orgánulos celulares clave en la producción de energía en forma de adenosín trifosfato (ATP). Adicionalmente están implicadas en otras funciones biológicas (10). La alteración de la función mitocondrial, ya sea hereditaria o adquirida (por mutaciones espontáneas del ADNmit o por toxicidad), dará lugar a diversos cuadros clínicos.

La toxicidad mitocondrial puede expresarse clínicamente de diferentes maneras, pero predomina la afección de tejidos altamente dependientes de la función mitocondrial (músculo esquelético y cardiaco, hígado, páncreas, etc.). Así mismo, cada ITIAN posee mayor afinidad por determinados tejidos y no siempre los mecanismos patogénicos están bien definidos, aunque la inhibición de la $\mathrm{ADN}$ polimerasa mitocondrial, que produce una alteración en la síntesis de las enzimas mitocondriales, parece ser el más aceptado (4). Los efectos adversos 
TABLA IV

\section{RELACIÓN ENTRE LOS DIFERENTES ITIAN Y LAS MANIFESTACIONES CLÍNICAS DE LA TOXICIDAD MITOCONDRIAL}

\begin{tabular}{ll}
\hline Efecto adverso & Fármacos inductores \\
\hline Hiperlactatemia y acidosis láctica & Todos, en especial: d4T, ZDV, ddl \\
Pancreatitis & ddd, ddC, d4T \\
Miopatía & ZDV \\
Neuropatía periférica & ddC, d4T, ddl \\
Mielotoxicidad & ZDV \\
Lipodistrofia & Todos, en especial, d4T \\
\hline
\end{tabular}

Modificada de Lozano F, Viciana P, ed. (3).

más graves relacionados con la toxicidad mitocondrial son la acidosis láctica y la pancreatitis.

La elevación de las cifras de lactato en sangre es el resultado de una alteración en el metabolismo mitocondrial del piruvato y representa el fracaso energético de la célula (10). La hipótesis patogénica más aceptada afirma que el inicio del problema radica en la inhibición de la ADN polimerasa mitocondrial (polimerasa $\gamma$ ) en el hígado. Como consecuencia de la alteración de este enzima, necesaria para la replicación del ADN, no se lleva a cabo la síntesis enzimática para realizar el ciclo de Krebs y la fosforilación oxidativa (Fig. 1). Aunque los ITIAN son los antirretrovirales que más se relacionan con la producción de hiperlactatemia, no son los únicos.

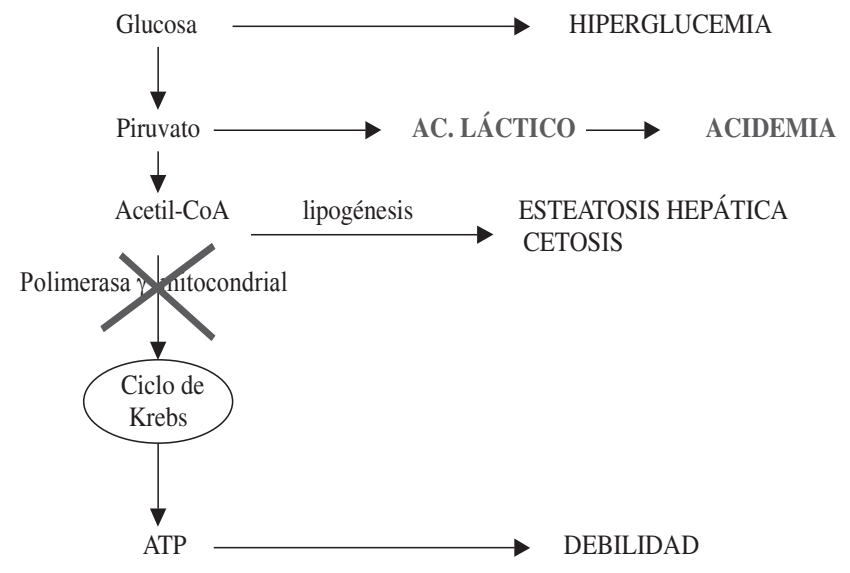

Fig. 1. Patogenia de la hiperlactatemia relacionada con IOS ITIAN. Modificada de Pulido F, ed. (12).

Se ha detectado hiperlactatemia leve en el $21 \%$ de los pacientes tratados con ITIAN, siendo la mayoría de ellas asintomáticas. La hiperlactatemia sintomática es menos común, su incidencia varía de 1,7 a 25,2 casos por 100 personas-año. Solo una minoría de pacientes desarrollan la forma más grave de hiperlactatemia, asociada a acidosis y a una elevada mortalidad (11). En general, el aumento de ácido láctico es un efecto adverso de los ITIAN a largo plazo, que suele aparecer tras varios meses de tratamiento.
En la revisión realizada por Falcó y cols., se vio que los ITIAN más frecuentemente implicados en hiperlactatemia fueron la estavudina $(48,3 \%)$ y la zidovudina $(45 \%)$ y las combinaciones más relacionadas con el desarrollo de acidosis láctica grave fueron estavudina-lamivudina y estavudinadidanosina (11). El menor riesgo estaría relacionado con el uso de abacavir y tenofovir (12).

No se conoce la causa, sin embargo las mujeres embarazadas tienen un riesgo mayor de hiperlactatemia y acidosis láctica (13). Según un estudio publicado en el año 2003 por Bonnet y cols., existen además dos factores que se relacionan con un riesgo elevado de desarrollo de acidosis láctica: el aclaramiento de creatinina $<70 \mathrm{~mL} / \mathrm{min}$ y un bajo recuento de CD4 (14). Sin embargo, la única variable asociada a la mortalidad son los niveles de ácido láctico en sangre $>10 \mathrm{mmol} / \mathrm{L}$ (11). Las manifestaciones clínicas suelen estar ausentes si las cifras de lactato no superan los $5 \mathrm{mmol} / \mathrm{L}$ (12). Cuando aparece sintomatología clínica, esta suele ser de carácter inespecífico: astenia, pérdida de peso (ocasionalmente aumento), náuseas, mialgias, dolor abdominal, disnea, etc. En la exploración física puede encontrarse hepatomegalia blanda, edema periférico, distensión abdominal, ascitis, encefalopatía, etc. En cuanto a los hallazgos analíticos es característica la aparición de aumento moderado de las transaminasas, hipoalbuminemia, hiperglucemia, hiperamilasemia, aumento del anión GAP, descenso del bicarbonato y en algunos casos acidosis. Las pruebas de imagen pueden evidenciar la existencia de una esteatosis hepática.

La determinación de los niveles de ácido láctico en sangre debe realizarse en condiciones adecuadas para evitar la presencia de falsos positivos y es necesario descartar otras posibles causas de hiperlactatemia (12). Dado que la hiperlactatemia leve-moderada asintomática es común, pero que raramente existe desarrollo de enfermedad grave, la determinación rutinaria de ácido láctico en pacientes tratados con ITIAN debe realizarse únicamente en el caso de mujeres embarazadas y pacientes en los que se reintroduce algún ITIAN tras un episodio de hiperlactatemia (13).

Dentro de las demás manifestaciones clínicas producidas por la toxicidad mitocondrial, la incidencia de pancreatitis se estima alrededor de un 4,7\%, pero es un efecto adverso dosis dependiente y se ha observado un aumento de la misma al incrementar la dosis de ddI (14). Los fármacos más relacionados con su aparición son la didanosina y el d4T. La incidencia de miopatía está relacionada con el uso de zidovudina (15). Es clínicamente difícil de distinguir de la miopatía producida por el VIH, sin embargo, histológicamente la producida por zidovudina presenta característicamente "ragged red fibers", que resultan de la acumulación subsarcolémica de las mitocondrias $(16,17)$. La zidovudina (aunque también otros ITIAN) puede producir alteraciones hematológicas como anemia, leucopenia y trombocitopenia. El mecanismo implicado puede ser la inhibición de la síntesis mitocondrial del grupo, probablemente por la inhibición de la ADN polimerasa $\gamma$ (18). Es necesario tener en cuenta la gran frecuencia con que se detecta macrocitosis en estos pacientes, especialmente en relación con análogos de timidina (ZDV, d4T). También se ha observado la aparición de una neuropatía axonal periférica en relación con el uso de ddI, d4T y ddC, que se caracteriza por la aparición de disestesias en dedos de manos y pies, disminución de los reflejos osteotendinosos, pérdida de sensibilidad a nivel distal y debilidad muscular moderada (10). Las concen- 
traciones de lactato en sangre podrían ser útiles para distinguir la neuropatía asociada al VIH, de la producida por los fármacos antirretrovirales (19). Por último, aunque la lipodistrofia se ha asociado principalmente con el uso de IP, también los ITIAN pueden producirla debido a la redistribución de la grasa corporal secundaria a las alteraciones mitocondriales (10). Sobre todo se ha asociado a la utilización de d4T y se manifiesta clínicamente como lipoatrofia.

El tratamiento de estas manifestaciones consiste básicamente en la retirada de los antirretrovirales. Esto, junto con el tratamiento sintomático necesario, que a veces incluye soporte en Unidades de Cuidados Intensivos, es la base del manejo terapéutico de la toxicidad mitocondrial. Al igual que en las enfermedades mitocondriales congénitas, se han realizado intentos de tratamiento con algunos fármacos como vitaminas esenciales, aceptores de electrones o L-carnitina, con resultados muy limitados (4).

La utilización del abacavir se relaciona con la aparición de reacciones de hipersensibilidad (RHS), las cuales constituyen su efecto adverso más característico y limitante de su uso. La mayor incidencia de RHS en relación con el abacavir se cifró en el $14 \%$ en un ensayo publicado en el año 2002 (20). La afectación cutánea no constituye el síntoma principal del cuadro clínico. Lo más característico y lo que ocasiona mayor gravedad, son los síntomas sistémicos (fiebre, náuseas/vómitos, artromialgias, cefalea, diarrea, dolor abdominal, disnea, etc.), que se instauran bruscamente, empeoran con la continuación del tratamiento y mejoran en pocos días tras la suspensión del mismo. Es necesario retirar el fármaco y en ningún caso reintroducirlo, ya que este hecho se relaciona con una rápida y muy grave reinstauración del cuadro de hipersensibilidad $(21,22)$.

Los ITIAN con menores efectos secundarios son 3TC y emtricitabina, que constituyen, por este motivo, la base de cualquier tratamiento nuevo en la actualidad.

INHIBIDORES DE LA TRANSCRIPTASA INVERSA NO ANÁLOGOS DE NUCLEÓSIDOS (ITINAN)

Los efectos adversos más frecuentemente producidos por los ITINAN son el exántema cutáneo por hipersensibilidad y las alteraciones del perfil hepático.

Los ITINAN son los antirretrovirales más relacionados con la aparición de alteraciones cutáneas por hipersensibilidad. Sin embargo, también el abacavir (ITIAN) y el amprenavir (IP) causan frecuentemente reacciones de este tipo. Son más frecuentes con nevirapina, pudiendo llegar a suponer una incidencia del 16-17\% en los pacientes tratados (23). Suelen aparecer tras 1-3 semanas de tratamiento en forma de una erupción eritematosa, maculopapulosa, pruriginosa y confluente, acompañada o no de manifestaciones constitucionales como fiebre, mialgias, artralgias, etc. En algunas ocasiones estas manifestaciones pueden estar presentes antes que las reacciones cutáneas o sin ellas (4). En menos de un 0,5\% de los casos pueden aparecer manifestaciones más graves, como síndrome de Stevens-Johnson, necrólisis epidérmica tóxica (4) o síndrome DRESS (drug rash, esosinophilia and systemic symptoms) (24). El diagnóstico se basa en la clínica, aunque en muchas ocasiones es difícil de diferenciar de otros procesos como la infección, la enfermedad maligna o la reconstitución inmunitaria, que hay que descartar (25). La patogenia no está definida, aunque se han sugerido múltiples mecanismos (4). Aproximadamente el 50\% de las RHS se resuelven de manera espontánea a pesar de mantener el tratamiento y únicamente debe retirarse ante la aparición de vesículas, exfoliación, disfunción hepática importante, afectación de mucosas, fiebre de $>39^{\circ}$ o prurito intenso (4). En el caso de la nevirapina, dada su mayor incidencia, se recomienda iniciar el tratamiento con una dosis baja, e incluso añadir antihistamínicos en los primeros días. En todos los casos de hipersensibilidad leve o moderada se puede probar una nueva exposición.

En cuanto a las alteraciones de la función hepática, todos los fármacos antirretrovirales pueden producirlas, especialmente cuando existen infecciones concomitantes por los virus de la hepatitis B o C y con alguna frecuencia conducen a la suspensión del tratamiento (26). La aparición de hepatitis es especialmente importante con nevirapina, siendo la incidencia del $1 \%$ en los pacientes tratados con dicho fármaco. En un $6 \%$ de los casos, se observa un aumento asintomático de los enzimas hepáticos (23). Se ha registrado una incidencia 12 veces superior de alteraciones hepáticas sintomáticas con el empleo de nevirapina en mujeres (entre ellas, las embarazadas) con recuentos de CD4 > 250 células $/ \mathrm{mm}^{3}$ previos al inicio de tratamiento antirretroviral. La incidencia también aumenta, aunque con menor intensidad, en varones con recuentos de CD4 > 400 células $/ \mathrm{mm}^{3}$ (27). La hepatotoxicidad puede ir desde una leve y asintomática alteración de las pruebas de función hepática, hasta un fallo hepático fulminante (28). Por todo ello, se recomienda que al prescribir nevirapina como tratamiento inicial en mujeres con recuentos de CD4 >250 células $/ \mathrm{mm}^{3}$ y en varones con CD4 > 400 células $/ \mathrm{mm}^{3}$, se realice una estrecha vigilancia de los síntomas clínicos y las alteraciones analíticas, especialmente durante los 3 primeros meses de tratamiento (27). Si se produce un aumento asintomático de los enzimas hepáticos 2 veces mayor a su valor basal, no se suspenderá el tratamiento, pero será necesaria vigilancia estricta. Si el aumento es 5 veces mayor, a pesar de la aparición o no de síntomas, se suspenderá el fármaco transitoriamente hasta que se normalicen los valores analíticos. Si tras reintroducir la nevirapina se produce un nuevo aumento de los enzimas hepáticos, será necesaria la suspensión permanente de la misma (directrices de la Agencia Europea del Medicamento, EMEA/11260/00) (29).

Es necesario destacar en este grupo, los efectos adversos específicos del efavirenz a nivel de la esfera neuro-psicológica. Este es el fármaco que presenta una mayor incidencia de reacciones adversas a nivel del sistema nervioso central (30). En un ensayo clínico realizado, se observó que el 52\% de los pacientes tratados sufrieron trastornos neuropsíquicos, pero sólo en el 2,6\% de los mismos fue necesario retirar el fármaco (31). Las principales manifestaciones clínicas son cefalea, pensamientos extraños, déficit de concentración, despersonalización, ansiedad, convulsiones, confusión, estupor, catatonia, depresión e incluso ideas suicidas. Suelen aparecer al inicio del tratamiento y desaparecer espontáneamente a las 2-4 semanas, por lo que no suele ser necesaria la suspensión del fármaco, pero sí la vigilancia estrecha sobre todo en aquellos pacientes con antecedentes de depresión u otras alteraciones mentales (30). Por otra parte, a partir de la comunicación de teratogenia en primates superiores, su uso está contraindicado (categoría D de la FDA) durante el embarazo. La contraindicación es especialmente importante en el primer trimestre, por lo que es un fármaco no aconsejable en mujeres que deseen tener un hijo (27). 


\section{INHIBIDORES DE LA PROTEASA (IP)}

En cuanto a los IP hay que destacar las alteraciones en el metabolismo lipídico e hidrocarbonado, así como el gran problema estético que supone la aparición de lipodistrofia, todos ellos efectos adversos tardíos. Tanto la dislipemia, como la lipodistrofia y la intolerancia a los hidratos de carbono, se pueden presentar asociados y tener un efecto negativo en el cumplimiento del tratamiento y en la calidad de vida de los pacientes (32). Se piensa además, que estos cambios metabólicos pueden producir en un futuro no muy lejano, una epidemia de enfermedades cardiovasculares en los pacientes sometidos al tratamiento antirretrovírico (4).

Los IP se han relacionado con un aumento de los niveles de triglicéridos y de colesterol total y LDL, disminuyendo el HDL colesterol $(33,34)$. Los más nuevos, como el atazanavir, producen menos efectos tóxicos metabólicos (34).

Aunque los mecanismos etiopatogénicos no son bien conocidos en la actualidad, se han propuesto diversas teorías tales como la regulación a la baja de los receptores de LDL en los monocitos, el aumento en la producción de lipoproteínas hepáticas y la regulación al alta de los receptores de HDL en los macrófagos (34-37). En la mayoría de los casos las alteraciones lipídicas no son importantes. Sin embargo, en los pacientes en los que son mayores y/o se asocian a otros factores de riesgo cardiovascular, es importante un diagnóstico y tratamiento precoz (32). Existen guías prácticas para el manejo terapéutico de las dislipemias en pacientes VIH (34). A la hora de su aplicación hay que tener en cuenta algunas consideraciones especiales (32): en primer lugar que en algunas ocasiones no se pueden poner en práctica las recomendaciones dietéticas debido a la delgadez e incluso caquexia padecida por algunos pacientes y en segundo lugar que los hipolipemiantes son hepatotóxicos, lo que supone un riesgo añadido en pacientes que en muchas ocasiones ya tienen una alteración hepática. En caso de decidir pautar tratamiento farmacológico, se aconseja elegir fibratos o estatinas, mejor pravastatina o atorvastatina por menor riesgo de interacciones medicamentosas. Será además necesario en estos casos, el control periódico de los enzimas de daño hepático.

Muy frecuentemente se ha objetivado resistencia a la insulina en pacientes tratados con IP. Hasta en un 15-40\% de ellos se ha descrito intolerancia a los hidratos de carbono y diabetes mellitus tipo 2 en un 8-10\% de los casos $(38,39)$. El mecanismo patogénico se desconoce, aunque parece estar relacionado con una inhibición de la captación celular de glucosa por parte de estos fármacos (40). Sin excluir un origen multifactorial, diversos estudios señalan que el bloqueo del GLUT-4, principal transportador de glucosa en el músculo y el adipocito, es el mecanismo fundamental responsable de las alteraciones del perfil glucémico producidas por los IP (41). Los resultados de los estudios que intentan relacionar las alteraciones hidrocarbonadas, con la distribución de la grasa corporal y los niveles de adipocitokinas, son aún dispares (41). En caso de que sea necesario su tratamiento, es importante evaluar las posibles interacciones farmacológicas con los medicamentos empleados en estos pacientes. Así, se desaconseja el uso de metformina porque puede aumentar el riesgo de hiperlactatemia y acidosis láctica (32) y en pacientes con alteración de los enzimas hepáticos, no se aconseja la utilización de tiazolidindionas por el riesgo de aumentar la hepatotoxicidad (42).
La lipodistrofia causada por el tratamiento antirretroviral, puede presentarse de dos formas clínicas diferentes, que pueden aparecer asociadas en un mismo paciente. La lipohipertrofia que se caracteriza por la acumulación de grasa central (en vísceras, abdomen, mamas y "cuello de búfalo") y la lipoatrofia que se define por la pérdida de grasa periférica (cara, glúteos y extremidades). Se ha objetivado que la aparición de lipodistrofia es más frecuente en los pacientes de mayor edad, con infección avanzada, con mejor respuesta al tratamiento antirretroviral, en mujeres y en pacientes de raza blanca (43). Los individuos delgados tienden a desarrollar lipoatrofia, mientras que los obesos lipohipertrofia (32). Aunque se han sugerido diversos mecanismos, no se conoce con exactitud la etiopatogenia de este proceso. Recientes estudios sugieren que los IP podrían producir una disminución de la adipogénesis y alteraciones en el aclaramiento lipídico. Algunos estudios sugieren que el tratamiento farmacológico de forma individual, no es suficiente para producir cambios en la redistribución de la grasa corporal y se han postulado a su vez, interacciones con citokinas y otros mediadores. En resumen, la patogenia de la la lipodistrofia parece obedecer a un origen multifactorial, aún no bien conocido (44). En la práctica diaria, la impresión clínica es la base del diagnóstico a pesar de la precisión de diversas pruebas como la tomografía o la resonancia magnética. El principal problema de la lipodistrofia es la gran implicación estética, y a solucionarlo es a donde se dirigen los mecanismos terapéuticos. En primer lugar se puede intentar el cambio de régimen de antirretrovirales por otro con menor efecto a este nivel. Otros procedimientos incluirían la cirugía plástica, la liposucción y los implantes de grasa autóloga u otros materiales sintéticos. Sin embargo, aunque se ha visto que los resultados a corto plazo son buenos, no se conocen las implicaciones a largo plazo (45).

En relación con la enfermedad cardiovascular en pacientes infectados por el VIH y en tratamiento antirretroviral, se han estudiado numerosos factores de riesgo: HTA, lipoproteína aLp(a), homocisteína, disfunción endotelial o ateroesclerosis. Aún no existen datos sobre la prevalencia de HTA en estos pacientes (34), en cambio si se ha observado que aproximadamente un $65 \%$ de sujetos infectados por el VIH, independientemente de recibir o no tratamiento antirretroviral, presentan cifras de lipoproteína a-Lp(a) elevadas (> $30 \mathrm{mg} / \mathrm{dL}$ ) (46). La homocisteinemia plasmática también podría ser hasta 3 veces superior en pacientes que reciben tratamiento (47). Se ha descrito una disfunción endotelial en pacientes con infección por el VIH, relacionada bien con el propio virus, con el tratamiento antirretroviral o con las alteraciones metabólicas secundarias al mismo (34). Algunos autores han puesto de manifiesto mediante ecografía doppler un aumento del grosor de la íntima-media arterial y la presencia de placas ateromatosas en pacientes en tratamiento antirretroviral, relacionando este con alteraciones a nivel de la pared vascular $(34,48)$. Por otra parte, se ha evidenciado una mayor prevalencia de isquemia miocárdica silente en sujetos que reciben IP con respecto a la población general, pero no se ha objetivado aún de forma clara una mayor frecuencia de síndromes coronarios agudos en estos pacientes $(34,49)$. En conclusión, es aún pronto para determinar si el tratamiento antirretroviral será responsable de un aumento de la morbimortalidad cardiovascular a largo plazo. Sin embargo, considerando la mayor prevalencia de factores de riesgo cardiovascular y de eventos cardiovasculares en estos pacientes respecto a la población general, es importante 
insistir en el diagnóstico y control precoz de los factores predisponentes. Así mismo, es de gran interés la elección del régimen terapéutico más adecuado y la posible aparición de interacciones medicamentosas (34).

Es importante conocer que los IP también producen efectos secundarios precoces con frecuencia, especialmente gastrointestinales, sobre todo diarrea con nelfinavir y lopinavir/ritonavir, y otros menos frecuentes como dolor abdominal, cefaleas, etc.

Finalmente destacar que existen efectos secundarios precoces específicos de alguno de estos fármacos, entre los que cabe destacar la nefrolitiasis por indinavir (hoy en día poco utilizado) y la hiperbilirrubinemia a expensas de bilirrubina indirecta, producida por este mismo fármaco y por atazanavir, que en raras ocasiones obliga a suspender el tratamiento.

\section{OTROS EFECTOS ADVERSOS DE LOS ANTIRRETROVIRALES}

Los inhibidores de la transcriptasa inversa no nucleótidos (tenofovir), aunque menos estudiados, se han asociado a la producción de acidosis tubular renal por un mecanismo de daño mitocondrial. No se recomienda su uso en pacientes con afectación de la función renal.

Otros procesos, como la osteonecrosis y la osteoporosis que aparecen de forma intensa y acelerada en algunos pacientes con infección por el VIH, se relacionan tanto con el propio virus, como con el tratamiento antirretroviral de gran actividad (50-52).

En la actualidad se dispone de un nuevo antirretroviral, enfuvirtida o T-20, perteneciente a un grupo farmacológico diferente, inhibidores de la fusión. Tiene pocos efectos secundarios, los más importantes derivados de problemas en el punto de inyección (nódulos cutáneos), debido a que debe utilizarse por vía subcutánea. Aunque poco frecuente, la neumonía bacteriana y las reacciones sistémicas de hipersensibilidad, también se han descritos en pacientes tratados con enfuvirtida (53).

\section{CONCLUSIONES}

La gran frecuencia, variedad y morbilidad de los efectos nocivos causados por los fármacos antirretrovirales, ha tenido importantes consecuencias sobre el balance riesgo-beneficio del TARGA, lo que ha ocasionado que las indicaciones para el inicio del tratamiento sean más restrictivas $(3,27,54-56)$. La prevención de los mismos y su diagnóstico precoz es fundamental en el seguimiento de estos pacientes. Ello implica el conocimiento de los mismos por parte del especialista, quien será responsable de informar al paciente de la posible aparición de los mismos y de iniciar, en caso de que sea necesario, un tratamiento rápido y certero (54-57).

\section{Bibliografía}

1. Palella FJ Jr, Chmiel JS, Moorman AC, Holmberg SD, and the HIV outpatient study investigators. Durability and predictors of success of highly active antiretroviral therapy for ambulatory HIV-infected patients. AIDS 2002; 16: 1617-26.

2. Mocroft A, Brettle R, Kirk O, Blaxhult A, Parkin, JM, Antunes F, et al. Changes in the cause of death among HIV positive subjects across Europe: results from the EuroSIDA study. AIDS 2002; 16: 1663-71.

3. Lozano F, Viciana P. Efectos adversos del tratamiento antirretrovírico: importancia y espectro clínico. En: Lozano F, Viciana P, editores. Efectos adversos del tratamiento antirretrovírico. Madrid: Scientific Communication Management, S.L.; 2003. p. 11-8.

4. Carr A, Cooper D. Adverse effects of antiretroviral therapy. Lancet 2000; 356: 1423-30.

5. Fellay J, Boubaker K, Ledergerber B, Bernasconi E, Furrer H, Battegay $\mathrm{M}$, et al. Prevalence of adverse events associated with potent antiretroviral treatment: Swiss HIV cohort study. Lancet 2001; 358: 1322-7.

6. Reisler RB, Han C, Burman WJ, Tedaldi EM and Neaton JD. Grade 4 events are as important as AIDS events in the era of HAART. J Acquir Immune Defic Syndr 2003; 34: 379-386.

7. Le Moing V, Chene G, Leport C, Lewden C, Duran S, Garré M et al. Impact of discontinuation of initial protease inhibitor therapy on further virological response in a cohort of human inmunodeficiency virus-infected patients. Clin Infect Dis 2002; 34: 239-47.

8. Dieleman JP, Jambroes M, Gyssens IC, Sturkenboom MCJM, Stricker B, Mulder WMC et al. Determinants of recurrent toxicity-driven switches of highly active antiretroviral therapy. The ATHENA cohort. AIDS 2002; 16; 737-45

9. Mocroft A, Youle M, Moore A, Sabin CA, Madge S, Cozzi Lepri A, et al. Reasons for modification and discontinuation of antiretrovirals: results for a single treatment centre. AIDS 2001; 15: 185-94.

10. Domingo P. Toxicidad mitocondrial. En: Lozano F, Viciana P, editores. Efectos adversos del tratamiento antirretrovírico. Madrid: Scientific Communication Management, S.L.; 2003. p. 19-45.

11. Falcó V, Rodríguez D, Ribera E, Martinez E, Miró JM, Domingo P, et al. Severe nucleoside-associated lactic acidosis in human inmunodeficiency virus-infected patients: report of 12 cases and review of the literature. Clin Infect Dis 2002: 34: 838-46.

12. Pulido F. Hiperlactatemia. En: Polo R. Coordinadora. Actualización en el tratamiento de la lipodistrofia y alteraciones metabólicas. Madrid: GlaxoSmithKline; 2003. p. 116-21.

13. Carr A. Lactic acidemia in infection with human inmunodeficiency virus. Clin Infect Dis 2003; 36 (Supl. 2): S96-100

14. Dassopoulos T, Ehrenpreis ED. Acute pancreatitis in human inmunodeficiency virus-infected patients: a review. Am J Med 1999; 107: 78-84.

15. Peters BS, Winer J, Landom DN. Mitochondrial myopathy associated with chronic zidovudine therapy in AIDS. Am J Med 1993; 86: 5-15.

16. Brown RH, Mendell JR. Distrofias musculares y otras enfermedades musculares. En: Kasper DL, Braunwald E, Fauci AS, Hauser SL, Longo DL, Jameson JL. Harrison, Principios de Medicina Interna. $16^{\mathrm{a}}$ ed. Madrid. McGraw-Hill-Interamericana; 2005. p. 2783-97.

17. Lewis W, Dalakas M. Mitochondrial toxicity of antiviral drugs. Nat Med 1995; 1: 417- 22.

18. Lutton JD, Mathew A, Levere RD, Abraham NG. Role of heme metabolism in AZT-induced bone marrow toxicity. Am J hematol 1990; 35: 15 .

19. Brew B, Tisch S, Law M. Lactate concentrations distinguish between nucleoside neuropathy and HIV distal symmetrical sensory polyneuropathy. Program and abstracts of the 8th Conference on Retroviruses and Opportunistics Infections; February 4-8, 2001; Chicago, Illinois, Abstract 9 .

20. Fallon J, Ait-Khaled M, Thomas DA, Brosgart CL, Eron Jr JJ, Feinberg $\mathrm{J}$, et al. HIV-1 genotype and phenotype correlate with virológical response to abacavir, amprenavir and efavirenz in treatment experienced patients. AIDS 2002; 16: 387-96.

21. Rivero A, Delgado R. Reacciones de hipersensibilidad. En: En: Lozano F, Viciana P, editores. Efectos adversos del tratamiento antirretrovírico. Madrid: Scientific Communication Management, S.L.; 2003. p. 63-87.

22. Hetherington S, McGuirk S, Powell G, Cutrell A, Naderer O, Spreen B, 
et al. Hypersensitivity reactions during therapy with the nucleoside reverse transcriptase inhibitor abacavir. Clin Ther 2001; 23: 1603-14.

23. Encinas de la Iglesia J, Pérez García ML, Fuertes Martín A. Efectos secundarios del tratamiento antirretroviral. FMC 2002; 9: 343-9.

24. Alonso Claudio G, Fuertes Martín A, De Dios Perrino S, Arévalo Velasco A. DRESS syndrome associated with nevirapine theraphy. Arch Intern Med 2001; 161: 2501-2.

25. Race EM, Adelson-Mitty J, Kriegel GR, Barlam TF, Reimann KA, Letvin NL, Japour AJ. Focal mycobacterial lymphadenitis following initiation of protease-inhibitor therapy in patients with advanced HIV-1 disease. Lancet 1998; 351: 252-5.

26. Núñez M, Soriano V. Hepatotoxicidad del tratamiento antirretroviral En: F. Lozano, P. Viciana, editores. Efectos adversos del tratamiento antirretrovírico. 1ed. Madrid: Roche Farma; 2003. p. 47-62.

27. Updated Adult Guidelines. October 6, 2005 (http: //aidsinfo.nih. gov/guidelines).

28. ClarK S, Creighton S, Portmann B, Taylor C, Wendon J, Cramp M Acute liver failure associated with antirretroviral treatment for VIH: a report of six cases. J Hepatol 2002; 36: 295-301.

29. Arribas JR, Gonzalez J. Manejo de los efectos secundarios frecuentes del tratamiento antirretroviral en adultos. En: González J, Moreno S, Rubio R, editores. Infección por VIH 2000. Madrid: Doyma, 2001; p. 241-75.

30. Corzo JE, Gómez JM. Otros efectos adversos del tratamiento antirretrovírico. En: Lozano F, Viciana P, editores. Efectos adversos del tratamiento antirretrovírico. Madrid: Scientific Communication Management, S.L.; 2003. p. 247-54.

31. Staszewski S, Morales-Ramírez J, Tashima KT. Efavirenz plus zidovudine and lamivudine, efavirenz plus indinavir, and indinavir plus zidovudine and lamivudine in the treatment oh HIV-1 infection in adults. Study 006 Team. N Engl J Med 1999; 341: 1865-73.

32. Roca B. Transtornos metabólicos relacionados con el VIH y el tratamiento antirretroviral. An. Med. Interna 2003; 20 (11): 585-93

33. Cheseaux JJ, Jotterand V, Aebi C, Gnehm H, Kind C, Nadal D, et al. Hy- perlipidemia in HIV-infected children treated with protease inhibitors: relevance for cardiovascular diseases. J Acquir Immune Defic Syndr 2002; 30: 288-93.

34. San Martín M. HIV and HAART: cardiovascular repercusión. Nutr Metabol Disord HIV Infect 2002; 1: 5-13.

35. Petit JM, Duong M, Florentin E, Duvillard L, Lizard G, Gambert M, et al. Down expresión of LDL receptor in HIV-infected patients on HAART: Quantification by flow cytometry. Program and abstract of the 8 th Conference on Retroviruses and Opportunistics Infections; February 4-8, 2001; Chicago, Illinois, Abstract 661.

36. Riddle TM, Kuhel DG, Woolwtt LA, Fichtenbaum C, Hui DY. HIV protease inhibitor therapy increases hepatic lipoprotein production via stabilization of activated sterol regulatory element-binding protein-1 (SREBP-1) in the nucleus. Program and abstracts of the 8th Conference on Retroviruses and Opportunistics Infections; February 4-8, 2001; Chicago, Illinois, Abstract 659.

37. Smart EJ, Mateev S, Kincer J, Greenberg R,N. Protease inhibitors upregulate SR-BI and HDL-dependent colesterol uptake in human macrophages: amechanism that may enhance foam cell formation. Program and abstracts of the 8th Conference on Retroviruses and Opportunistics Infections; February 4-8, 2001; Chicago, Illinois, Abstract 662.

38. Hadigan C, Meigs JB, Corcoran C, Rietschel P, Piecuch S, Basgoz N, et al. Metabolic abnormalities and cardiovascular disease risk factors in adults with human inmunodeficiency virus infection and lipodystrophy. Clin Infect Dis 2001; 32: 130-9.

39. Martínez E, García MA, Conget I, Buira E, Blanco JL, Mallolas J, et al.
Incidence, characteristics, and prognosis of diabetes mellitus associated with protease inhibitors. 7th Conference on Retroviruses and Opportunistics Infections. San Francisco, 2000. Abstract 16.

40. Miserez AR, Muller PY, Spaniol V. Indinavir inhibits sterolregulatory element-binding protein-1c-dependent lipoprotein lipase and fatty acid synthase gene activations. AIDS 2002; 16: 1587-94.

41. Miralles C. Insuline resístanse in HIV patients: pathogenic role of dyslipidaemia and body fat redistribution. En: Nutrition and Metabolic Disorders in HIV infection, 2003; 2: 79-97.

42. O'Moore-Sullivan TM, Prins JB. Thiazolidinediones and type 2 diabetes: new drugs for an old disease. Med J Aust 2002; 176: 381-6.

43. Lichtenstein KA, Ward DJ, Moorman AC, Delaney KM, Young B Palella FJ Jr. Clinical assessment of HIV-associated lipodystrophy in an ambulatory population. AIDS 2001; 15: 1389-98.

44. Koutkia P and Grinspoon S. HIV-associated lipodystrophy: patogénesis, prognosis, treatment and controversies. Annu Rev Med 2004; 55: 30317.

45. Engelson ES, Glesby MJ, Mendez D, Albu JB, Wang J, Heymsfield SB, Kotler DP. Effect of recombinant human growth hormone in the treatment of visceral fat accumulation in HIV infection. J Acquir Immune Defic Synd 2002; 30: 379-91.

46. Koppel K, Bratt G, Eriksson M, Sandstrom E. Serum lipid levels associated wiyh increased risk for cardiovascular disease is associated with highly active antirretroviral therapy (HAART) in HIV-1 infection. Int J STD AIDS 2000; 11: 451-55.

47. Bernasconi E, Uhr M, Telenti M, Bernasconi A, Magenta L, Moccetti T. Homocysteinemia in HIV-1 infected patients treated with HAART. Program and abstracts of the Seventh European Conference on Clinical Aspectects and Treatment of HIV-Infection, Lisbon, Portugal, Octubre 23-27, 1999. Abstract 806

48. Maggi P, Serio G, Epifani G, Fiorentino G, Saracino A, Fico C, et al. Premature lesions of the carotid vessels in HIV-1 infected patients treated with protease inhibitors. AIDS 2000, 14: 123-8.

49. Doung M, Cottin Y, Piroth L, Fargeot A, Lhuiller I, Bobillier M et al. Exercise stress testing detection of silent myocardial ischemia in human inmunodeficiency virus-infected patients receiving antirretroviral therapy. Clinical Infectious Diseases 2002; 34: 523-8.

50. Miller KD, Masur H, Jones EC, Joe GO, Rick ME, Kelly GG et al. High prevalence of osteonecrosis of the femoral head in HIV-infectede adults. Ann Intern Med 2002; 137: 17-25.

51. Blacksin MF, Kloser PC, Simon J. Avascular necrosis of bone in human inmunodeficiency virus infected patients. Clin Imaging 1999; 23: 314-8.

52. Tebas P, Powderly WG, Claxton S, Marin D, Tantisiriwat W, Teitelbaum SL, et al. Accelerated bone mineral loss in HIV infected patients receiving potent antiretroviral therapy. AIDS 2000; 14: F63-7

53. Clotet B, Raffi F, Cooper D, Delfraissy JF, Lazzarin A, Moyle G, et al. Clinical management of treatment-experienced, HIV-infected patients with the fusion inhibitor enfuvirtide: consensus recommendations. AIDS 2004, 18: 1137-46.

54. Henry K. The case for more cautious, patient-focused antiretroviral therapy. Ann Intern Med 2000; 132: 306-11.

55. Tebas P. On antiretroviral guidelines and evidence based medicine. Enferm Infecc Microbiol Clin 2002; 20: 241-3.

56. Rubio R, Berenguer J, Miró JM, Antela A, Iribarren JA, González J, et al. Recomendaciones de GESIDA/Plan Nacional sobre SIDA respecto al tratamiento antirretroviral en pacientes adultos infectados por el virus de la inmunodeficiencia humana en el año 2002. Enferm Infecc Microbiol Clin 2002; 20: 244-303.

57. Max B, Sherer R. Management of de adverse effects of antiretroviral therapy and medication adherence. Clin Infect Dis 2000; (Supl. 2): S96-116. 